\title{
Paradigma do Acesso Aberto (Open Access): alguns apontamentos para os estudiosos do turismo no Brasil
}

\author{
Open Access Paradigm: some notes to tourism studious in Brazil
}

Cristina Marques Gomes ${ }^{1}$

\section{Resumo}

O presente artigo versa sobre o Paradigma do Acesso Aberto (ou Open Access - OA) traçando um panorama geral sobre os movimentos e iniciativas, como e em quais contextos os mesmos surgiram, quais são os conceitos envolvidos nos debates em torno da temática, as linhas de atuação, os principais manifestos, argumentações teóricas, pesquisadores, dentre outros aspectos, com o objetivo de contribuir para debates ulteriores sobre as interfaces da Ciência da Informação e do Turismo. Apresenta os sub-itens "Acesso Aberto (Open Access): Conceitos, Movimentos e Iniciativas" e "Vias Verde e Dourada", culminando com as Considerações Finais sobre o tema.

Palavras-Chave: turismo; acesso aberto; Vias Verde e Dourada.

\begin{abstract}
This article is about the Open Access Paradigm (or just AO), delineating a general panorama about movements and enterprises, how and in which contexts they arised, which are the involved concepts in discussion about the issue, the actuation lines, the main manifests, the theoretical argumentation, the researcheres, among other aspects, holping to contribute to ulterior discussion about Information Science and Tourism interfaces. Features the secondary items "Open Access: Concepts, Movements and initiatives" and Green and Golden Road, culminating to the Final Considerations about the issue.
\end{abstract}

Keywords: tourism; open access; Green and Golden Road.

\footnotetext{
${ }^{1}$ Doutoranda do Programa de Tecnologias e Sistemas de Informação (PDTSI) da Universidade do Minho (Portugal); Doutoranda em Ciência da Informação - Escola de Comunicações e Artes da Universidade de São Paulo (ECA/USP - Brasil); e Docente da Universidade Federal do Estado do Rio de Janeiro (UNIRIO - Brasil). Email: cristina@usp.br.
} 


\section{Introdução}

O Turismo enquanto objeto de estudo em âmbito nacional emerge na década de 1970 com a criação das primeiras Instituições de Ensino Superior e as primeiras defesas de dissertações e teses sobre o tema em múltiplos Programas de Pós-Graduação. Em sua pequena trajetória temporal muitos debates ocorreram em torno da "interdisciplinaridade" da área e suas interfaces com outras disciplinas. Mas até que ponto esse "intercâmbio" e/ou "troca de saberes" acontece? Quando adentramos uma área extremamente abrangente como, por exemplo, a Ciência da Informação (CI) constatamos, inclusive a partir de uma pesquisa com amostragem aleatória realizada em 2009, que os estudiosos do Turismo muito pouco ou nada conhecem sobre uma série de teorias e/ou linhas de pesquisa que estão diretamente relacionadas com o caráter epistemológico de uma possível "Ciência do Turismo". Nesse sentido, e com o intuito de contribuir para debates ulteriores sobre as entrelinhas entre as duas áreas (CI e Turismo), o presente artigo versará sobre o Paradigma do Acesso Aberto (ou Open Access - $O A$ ) traçando um panorama geral sobre os movimentos e iniciativas, como e em quais contextos os mesmos surgiram, quais são os conceitos envolvidos nos debates em torno da temática, as linhas de atuação, os principais manifestos, argumentações teóricas, pesquisadores, etc.

A justificativa para tal empreitada, também, está sustentada no reduzido número de publicações, no Brasil e no exterior, sobre as interfaces do OA com o Turismo. A título de ilustração citamos um trabalho de nossa autoria intitulado "Comunicação Científica, Acesso Aberto (Open Access) e Novas Tecnologias na Contemporaneidade: Cartografia das Vias Dourada e Verde na Ciência do Turismo" publicado na Revista Turismo \& Desenvolvimento (Journal of Tourism and Development) da Universidade de Aveiro (Portugal) e o artigo do Bing Pan do Department of Hospitality and Tourism Management do College of Charleston (USP) - "A Model of Scholarly Communication in Tourism and an Open Access Initiative".

Esclarecemos, ainda, que além dessa introdução, esse texto contará com os sub-itens: Acesso Aberto (Open Access): Conceitos, Movimentos e Iniciativas; Vias Verde e Dourada; e as Considerações Finais sobre o tema. 


\section{Acesso Aberto (Open Access): conceitos, movimentos e iniciativas}

De início, salientamos que Open Access (OA), ou seja, Acesso Aberto é uma expressão contemporânea que nos remete, além do significado que o termo encerra, a um movimento político que busca alterar o modelo de negócios vigente em relação aos Periódicos Científicos rompendo-se, em parte, com o sistema tradicional da Comunicação Científica via, e graças, ao desenvolvimento das Novas Tecnologias. As nuances do verbete tangenciam as exprimidas pelo "Acesso Livre" e é importante perceber as tênues diferenças entre o "grátis" e o "livre", esbarrando, inclusive, nos softwares livres que emergem com o intuito de serem modificados por qualquer pessoa e na questão dos significantes em diferentes contextos idiomáticos e culturais.

Conceitualmente, evidenciam-se as definições proferidas por Stevan Harnad ${ }^{2}$ para o qual Open Access "is free, immediate, permanent online access to the full text of research articles for anyone, webwide" e Peter Suber ${ }^{3}$ - "Open Access literature is digital, online, free of charge, and free of most copyright and licensing restrictions". Esses dois autores compõem com mais 14 outros $^{4}$ o grupo que atestou o Movimento de Acesso Livre (ALi) a partir de dois documentos principais: a Declaração de Budapeste (Budapest Open Access Initiative) em fevereiro de 2002, convocada pelo Programa de Informação do Open Society Institute (OSI) e a Declaração de Berlim (Berlin Declaration) do ano seguinte. Ambas descritas ulteriormente.

Em âmbito nacional, Lara (2006) em seu Glossário de Termos e Conceitos da Área de Comunicação e Produção Científica, define OA, com base na Declaração de Budapeste, como:

(...) disponibilização livre na Internet de literatura de caráter acadêmico ou científico (em particular os artigos de revistas científicas), permitindo a qualquer

\footnotetext{
${ }^{2}$ HARNAD, Stevan. (s.d.) Open Access. Disponível em: <http://www.eprints.org/openaccess/>, acessado em 3 de abril de 2009.

${ }^{3}$ SUBER, Peter. 2007. Open Access Overview: Focusing on open acces to peer reviewd research articles and their preprints. Disponível em: <http:/www.earlham.edu/ peters/fos/overview.htm>, acessado em 3 de abril de 2009.

${ }^{4}$ Leslie Chan (Bioline International), Michael Eisen (Public Library of Science), Fred Friend (University College London), Yana Genova (Next Page Foundation), Jean-Claude Guédon (University of Montreal), Rick Johnson (Scholarly Publishing and Academic Resources Coalition), Manfredi La Manna (Electronic Society for Social Scientists), Monika Segbert (eIFL Project), Sidnei de Souza (CRIA, Bioline International), Jan Velterop (BioMed Central) e quatro representante do Open Society Institute, vinculado a fundação Soros: Darius Cuplinskas, Melissa Hagemann,Rima Kupryte e István Rév.
} 
utilizador ler, baixar arquivos, copiar, distribuir, imprimir, pesquisar, indexar, fazer links ou referenciar o texto integral dos documentos. A única restrição sobre a reprodução e distribuição é o direito de ser reconhecido e citado, cabendo aos autores o controle sobre a integridade dos trabalhos. A iniciativa [...] pode significar a eliminação de barreiras de acesso à literatura científica, permitindo acelerar a pesquisa e incentivar o diálogo intelectual para o conhecimento.

E, no "Manifesto Brasileiro de Apoio ao Acesso Livre à Informação Científica" temos que:

I. Contribuições em acesso livre incluem resultados de pesquisas científicas originais, dados não processados, metadados, fontes originais, representações digitais de materiais pictóricos, gráficos e material acadêmico multimídia.

II. As contribuições em acesso livre devem satisfazer duas condições:

1. os(s) autor(es) e o(s) detentores dos direitos de tais contribuições concede(m) a todos os usuários: a. direito gratuito, irrevogável e irrestrito de acessá-las; b. licença para copiá-las, usá-las, distribuí-las, transmiti-las e exibi-las publicamente; c. licença para realizar e distribuir obras derivadas, em qualquer suporte digital para qualquer propósito responsável, em obediência à correta atribuição da autoria (as regras da comunidade continuarão a fornecer mecanismos para impor a atribuição e uso responsável dos trabalhos publicados, como acontece no presente) e com a garantia de fazer cópias;

2. Uma versão completa da obra e todos os materiais suplementares, incluindo uma cópia da licença, como acima definida, é depositada e, portanto, publicada em um formato eletrônico normalizado e apropriado em pelo menos um repositório que utilize normas técnicas adequadas (como as definições estabelecidas pelo modelo Open Archives) e que seja mantido por uma instituição acadêmica, sociedade científica, organismo governamental, ou outra organização estabelecida que pretenda promover o acesso livre, a distribuição irrestrita, a interoperabilidade e o arquivamento a longo prazo 5 .

A expressão "interoperabilidade" (inter- + operabilidade), citada no Manifesto acima, é a qualidade do que é interoperável, ou seja, a capacidade de um sistema para interagir e comunicar com outro. Em termos técnicos podemos caracteriza-lá como: um conjunto mínimo de $\operatorname{metadados}{ }^{6}$; a concordância no uso de uma sintaxe $\mathrm{XML}^{7}$ para transportar e

\footnotetext{
${ }^{5}$ Manifesto Brasileiro de Apoio ao Acesso Livre à Informação Científica. 2008. Disponível em: Disponível em: $<\mathrm{http} / /$ kuramoto.files.wordpress.com/2008/09/manifesto-sobre-o-acesso-livre-a-informacao-cientifica.pdf>, acessado em 11 de outubro de 2009.

${ }^{6}$ Os metadados são informação que resume, enriquece ou complementa os objetos ou serviços referenciados, produzindo assim um potencial "incremento de informação".
} 
representar dados; e a definição de um protocolo comum para extrair os mesmos ${ }^{8}$. A "interoperabilidade" juntamente com o "auto-arquivamento"9 e a "revisão pela comunidade" constituem os princípios básicos do conceito de "arquivos abertos" (open archives) definido durante a Convenção de Santa Fé ${ }^{10}$ em 1999 realizada no Novo México, com o apoio da Council on Library and Information Resources (CLIR), da Digital Library Federation (DLF), da Scholarly Publishing \& Academic Resources Coalition (SPARC), da Association of Research Libraries (ARL) e do Los Alamos National Laboratory (LANL).

Salienta-se que, na "prática", temos duas iniciativas distintas, de um lado a Open Access Initiative (OAI) que emerge com a Convenção supracitada e, de outro, o Movimento de Acesso Livre (ALi) que advém da Declaração de Budapeste, ambos, no entanto, convergem para o mesmo propósito, ou seja, o Acesso Aberto (WEITZEL, 2006).

Em trechos de uma entrevista dada por Steven Harnad com versão ao Português por Hélio Kuramoto na Revista Ciência da Informação ${ }^{11}$ de 2007, o pesquisador afirma que é importante não confundirmos os arquivos abertos, ou seja, interoperáveis, em conformidade com o modelo estabelecido pela OAI, com arquivos do Movimento do Acesso Livre (ALi): um arquivo pode estar em conformidade com os padrões estabelecidos pela OAI, mas não ser de ALi e vice-versa. E, ainda, que não existe uma correspondência direta do ALi com o software livre ou Open Source ${ }^{12}$ e nem com a licença Creative Commons ${ }^{13}$. Para o

\footnotetext{
${ }^{7}$ Linguagem genérica de descrição estrutural de documentos digitais.

${ }^{8}$ Os mecanismos para estabelecer a Interoperabilidade descritos no artigo de Sompel \& Lagoze (2000) e detalhados na Convenção são: 1. The definition of a set of simple metadata elements - the Open Archives Metadata Set (OAMS) - http://www.openarchives.org/sfc/sfc_oams.htm - for the sole purpose of enabling coarse granularity document discovery among archives; 2. The agreement to use a common syntax, XML, for representing and transporting both the OAMS and archive-specific metadata sets; 3 . The definition of a common protocol - the Open Archives Dienst Subset - http://www.cs.cornell.edu/cdlrg/dienst/protocols/OpenArchives Dienst.htm - to enable extraction of OAMS and archive-specific metadata from participating archives.

9 “'Auto-arquivamento ou self-archiving é um mecanismo que permite aos próprios autores submeter ou depositar os seus trabalhos ou papers em um repositório digital" (Kuramoto, 2006, p.94).

${ }^{10}$ The Santa Fe Convention for the Open Archives Initiative. 1999. Disponível em: $<\mathrm{http}: / /$ www.openarchives.org/sfc/sfc_entry.htm>, acessado em 10 de setembro de 2009.

${ }^{11}$ Revista Ciência da Informação. Disponível em: <http://revista.ibict.br/ciinf/index.php/ciinf>, acessado em 2 de setembro de 2009.

12“Open Source" ou "Código Aberto" é uma iniciativa da "Open Source Initiative" (disponível em: $<$ http://www.opensource.org/>, acessado em 15 de outubro de 2009) e faz referência ao "Software Livre". Este, por sua vez, é assim designado em função do cumprimento de 4 "variáveis de liberdade" definidas pela "Free Software Fundation" (disponível em: <http://www.fsf.org/>, acessado em 15 de outubro de 2009).

13 "Creative Commons" designa, nesse caso, o conjunto de licenças padronizadas para a gestão livre e compartilhada do conhecimento científico e não a Organização Sem Fins Lucrativos norte-americana homônima. Esta, inclusive, mantém atualizada as discussões sobre a primeira.
} 
pesquisador: "o ALi aplica-se principalmente a artigos, à exceção de revistas gratuitas, todos aqueles que o autor escreveu somente para efeito de maximizar o seu uso e impacto, sem finalidade lucrativa, mas cujos copyrights foram gratuitamente entregues aos editores"14.

Nessa perspectiva de análise é fundamental citarmos o artigo "The Santa Fe Convention of the Open Archives Initiative ${ }^{, 15}$ publicado em fevereiro de 2000 na D-Lib Magazine por Herbert Van de Sompel ${ }^{16}$ e Carl Lagoze ${ }^{17}$. Nele os autores descrevem que a origem da iniciativa dos arquivos abertos está diretamente relacionada com o número crescente de "electronic preprint (e-print) archives". Os arquivos de e-prints ${ }^{18}$ começaram a ser disseminados de forma informal com o objetivo de divulgar os resultados preliminares e não-revisados da literatura cinzenta $^{19}$ e evoluíram para um sistema de partilha de pesquisas entre colegas de determinada área, modificando, por consequência, o modelo tradicional de comunicação científica a medida que os textos eram disponibilizados antes da avaliação por pares.

Esclarece-se que a fase intitulada de "pré-publicação" acontece quando a pesquisa está em elaboração e o autor dialoga com seus colegas de forma informal ou via congressos, etc, com o propósito de lapidar o manuscrito e, aí sim, submetê-lo aos periódicos tradicionais obtendo, em seguida, o devido "parecer por pares". Além disso, outros feedbacks podem ser agregados ao processo na "pós-publicação" sob a forma de "open peer commentary" ${ }^{20}$. No entanto,

\footnotetext{
${ }^{14}$ Entrevista com Steven Harnad (versão Hélio Kuramoto). In: In: Bibli.: R. Eletr. Bibliotecon. Ci. Inf., Florianópolis, n. Esp., $1^{\mathrm{o}}$ sem. 2007. Disponível em: <http://www.periodicos.ufsc.br/index.php/eb/article /viewFile/647/518>, acessado em 1 de junho de 2009.

${ }^{15}$ SOMPEL, Herbert van de; LAGOZE, Carl. The Santa Fé Convention of the Open Archives Initiative. D-Lib Magazine, v. 6, n. 2, Feb. 2000. Disponível em: <http://www.dlib.org/dlib/february00/vandesompeloai/02vandesompel-oai.html>, acessado em 10 de setembro de 2009.

${ }^{16}$ Los Alamos National Laboratory - Research Library, New Mexico, US, and Automation Department of the Central Library of the University of Ghent, Belgium. herbert.vandesompel@rug.ac.be.

${ }^{17}$ Department of Computer Science. Cornell University. lagoze@cs.cornell.edu.

${ }^{18}$ Os repositórios digitais, uma forma de alternativa ao tradicional sistema de comunicação científica, muitas vezes também são denominados de "e-prints".

19،A Quarta Conferência Internacional sobre Literatura Cinzenta (GL'99), realizada em Washington, DC, em outubro de 1999, definiu literatura cinzenta como: "O que é produzido em todos os níveis do governo, institutos, academias, empresas e indústria, em formato impresso e eletrônico, mas que não é controlado por editores científicos ou comerciais." Em geral, considera-se literatura cinzenta publicações não-convencionais, evasivas e, às vezes, efêmeras. Podem incluir, mas não estão limitadas aos seguintes tipos de materiais: relatórios (préimpresso, preliminar e avançados, técnicos, relatórios estatísticos, memorandos, estudos de mercado, etc), Teses, actas de conferências, especificações técnicas e normas, traduções não-comerciais, bibliografias, documentação técnica e comercial, bem como documentos oficiais não publicados comercialmente (principalmente relatórios de governo e documentos) (Alberani, 1990)". disponível em: <http://ses.sp.bvs.br/local/File/literatura\%20 cinzenta_trad.pdf $>$, acessado em 19 de outubro de 2009.

${ }^{20}$ HARNAD, Stevan. 1990. Scholarly Skywriting and the Prepublication Continuum of Scientific Inquiry. Disponível em: <http://cogprints.org/1581/0/harnad90.skywriting.html>, acessado em 12 de fevereiro de 2009.
} 
“comentários pelos pares" (peer commentary) não substituem a avaliação prévia pelos pares $\left(\right.$ peer review) ${ }^{21}$.

O primeiro repositório ${ }^{22}$ de documentos eletrônicos com o princípio dos arquivos abertos é o ArXiv (http://arxiv.org/) criado em 1991 pelo físico Paul Ginsparg do Laboratório de Los Alamos no Novo México. Nele os pesquisadores localizados geograficamente em qualquer lugar do mundo enviavam seus trabalhos, muitas vezes ainda não revisados pelos pares ${ }^{23}$, para "uma central" que possibilitava a recuperação dos mesmos por quaisquer outros autoresassinantes. Peter Lepage (citado por BUTLER; 2001) na seção Debates da Revista Nature ${ }^{24}$, quando da saída de Ginsparg do Laboratório citado acima para a Universidade de Cornell no Estado de Nova York, comenta que o mesmo "completely transformed the nature and reach of scientific information in physics and other fields". Percebe-se, pois, que a interface técnica e/ou tecnológica foi alavancada a priori dos manifestos e da "conscientização" do ALi em função, principalmente, da "tradição" dos cientistas da área da física em partilhar os seus documentos (pré-prints). Além do ArXiv, Sompel \& Lagoze (2000) citam como relevantes as seguintes iniciativas:

- CogPrints (http://cogprints.soton.ac.uk/) - localizado na Universidade de Southampton no Reino Unido. Segue o modelo do ArXiv e usa o software e.print. Abrange as áreas de psicologia, lingüística, neurociências, ciência da computação, filosofia e biologia.

- NCSTRL (http://www.ncstrl.org/) (Network Computer Science Technical Reference Library) - coleção internacional sobre relatórios de pesquisa em ciência da computação. É uma rede construída segundo um modelo descentralizado. Os documentos são armazenados em repositórios distribuídos e disponibilizados por serviços também distribuídos, via protocolo Dienst.

- NDLTD (http://www.ndltd.org/) (Network Digital Library Thesis and Dissertations) - biblioteca eletrônica de teses e dissertações autorizadas por estudantes das instituições membros da rede. As pesquisas desenvolvidas para a criação desta rede envolvem assuntos como a criação de um fluxo (workflow) para

\footnotetext{
${ }^{21}$ HARNAD, Steven. 1998. Learned inquiry and the net: the role of peer review, peer commentary and copyright. Disponível em: <http://eprints.ecs.soton.ac.uk/2633/2/harnad98.toronto.learnedpub.html>, acessado em $12 \mathrm{de}$ fevereiro de 2009.

${ }^{22}$ Repositório: Lugar onde se deposita ou guarda alguma coisa. Dicionário Priberam da Língua Portuguesa. Disponível em: < http://www.priberam.pt>, acessado em 2 de fevereiro de 2009.

23 O próprio sistema checava a "qualidade mínima" dos textos, por exemplo, verificando a filiação do autor. ${ }^{24}$ BUTLER, Declan. 2001. Disponível em: <http://www.nature.com/nature/debates/eaccess/Articles/ginsparg.html>, acessado em 17 de agosto de 2009.
} 
submissão de ETD (Electronic Theses and Dissertations), o desenvolvimento de XML e de DTD (Document Type Definition) para ETDs e o suporte para a biblioteca digital de teses e dissertações eletrônicas.

- RePEc (http://www.ndltd.org/) (Research Papers in Economics) atua na área de economia e foi desenvolvida seguindo um modelo distribuído. Segue o protocolo Guilford, que garante a interoperabilidade entre os arquivos da $\mathrm{RePEc}^{25}$.

Ainda com base em Sompel \& Lagoze (2000) temos uma série de projetos/pesquisas que ilustram modelos alternativos de Comunicação Científica. Tais propostas lidam com modelos centralizados institucionalmente ou distribuídos em departamentos, com trabalhos que tangenciam a literatura cinzenta ou são revisados por pares, dentre outras variáveis. Em todas elas, no entanto, o propósito primeiro é a maximização dos impactos científicos ocasionado, por conseguinte, mecanismos mais eficazes de comunicação científica. Interoperabilidade pode incluir, por ora, diversos formatos de metadados, serviços em bibliotecas digitais, capacidade de contribuir para um sistema de métricas para uso coletivo, etc (SOMPEL \& LAGOZE; 2000). Na descrição pormenorizada da Convenção de Santa Fé, Sompel \& Lagoze (2000) discorrem sobre as visões "pré-concebidas" dos participantes do encontro e a proposta de convergirem tais noções díspares em um acordo bem definido que fosse capaz de fornecer os alicerces para a "troca de arquivos e informações".

Para alcançar seus objetivos a Convenção de Santa Fé também instituiu dois elementos no seu respectivo modelo: os provedores de dados (data providers) e os provedores de serviços (service providers), assim descritos:

Os provedores de dados são os gestores de arquivos e-prints, os quais devem ser dotados, no mínimo, das seguintes funcionalidades:

- mecanismos de submissão para o auto-arquivamento dos trabalhos ou papers;

- sistema de armazenamento a longo prazo;

- mecanismos de exposição de metadados do arquivo para facilitar a sua colheita

${ }^{25}$ Tradução TRISKA, Ricardo; CAFÉ, Lígia. Arquivos abertos: subprojeto da Biblioteca Digital Brasileira. In: Ciência da Informação. vol.30 no.3 Brasília Sept./Dec. 2001. Disponível em: $<$ http://www.scielo.br/scielo.php?script=sci_arttext\&pid=S0100-19652001000300012>, acessado em 11 de outubro de 2009. 
por terceiros, ou provedores de serviços.

$[\ldots]$

Os provedores de serviços são as instituições ou serviços de terceiros que implementam os serviços com valor agregado a partir dos dados coletados junto aos arquivos e-prints, ou repositórios digitais (KURAMOTO, 2006, p.94).

Em termos técnicos, também, esclarece-se que:

[...] O modelo Open Archives estabeleceu um protocolo de comunicação para que se possibilite a realização da coleta de metadados. Esse protocolo denomina-se Open Archives Initiative Protocol for Metadata Harvesting. Trata-se de um protocolo de comunicação que possibilita a coleta de metadados a partir de determinado provedor de dados. O provedor de serviços, para realizar a coleta de metadados, deve utilizar um programa chamado Harvester (mecanismo de colheita), o qual implementa esse protocolo, o OAI-PMH. O Harvester, ao visitar um provedor de dados, dialoga com o programa desse provedor, o qual deve estar preparado para atender a uma demanda do Harvester e expor os metadados solicitados por esse mecanismo de colheita.

Os metadados devem também constituir um padrão. O padrão normalmente utilizado pelos mecanismos de colheita é o Dublin Core sem qualificadores. Esse padrão poderá ser adaptado (KURAMOTO, 2006, p.94).

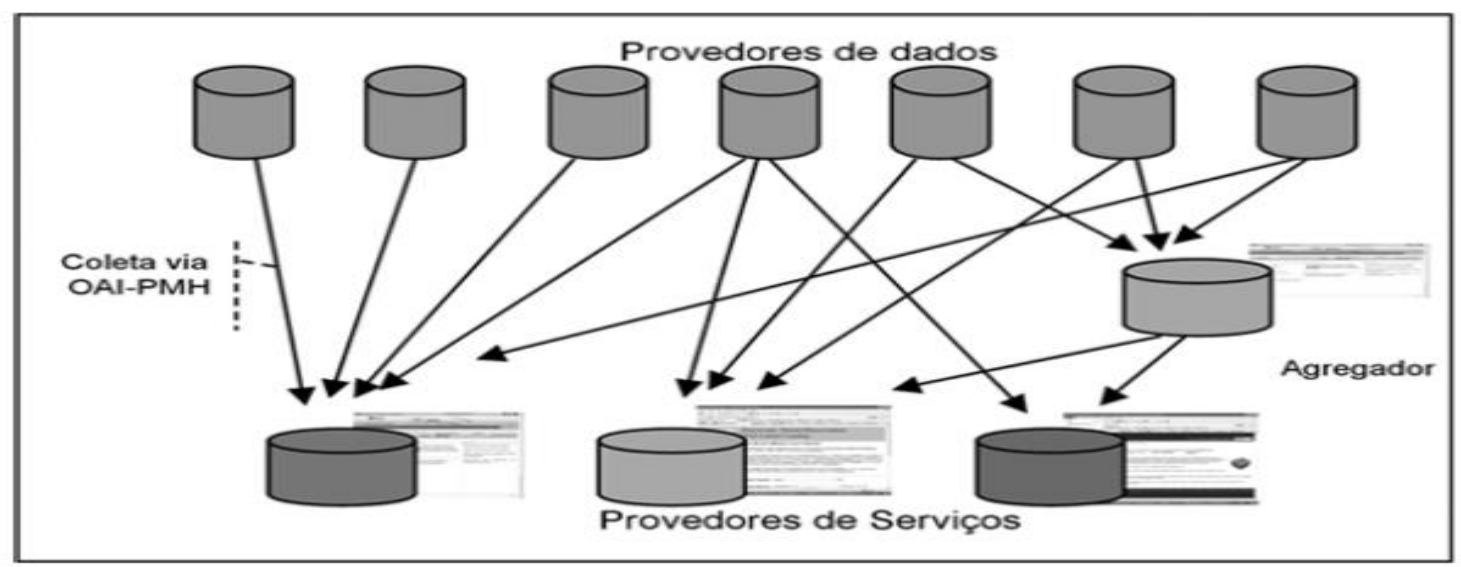

Figura 1 - Esquema Funcional do Modelo AO

Fonte: Kuramoto, 2006. 
Antes da Convenção de Santa e mesmo do surgimento do ArXiv, um outro "borbulhar" já se fazia presente com a conhecida "crise dos periódicos" em meados da década de 1980, quando as bibliotecas universitárias, principalmente americanas, não tinham mais "fôlego" para sustentar financeiramente a compra de novos periódicos científicos. O ciclo era ${ }^{26}$ : o pesquisador, subsidiado com dinheiro público, realizava sua pesquisa que era publicada em uma revista referenciada que, por sua vez, era vendida pela editora responsável para a biblioteca da mesma instituição que o autor em questão desenvolveu o seu trabalho, ou seja, "pagava-se, com dinheiro público, duas vezes pela mesma pesquisa”. O debate em torno dessa temática é pertinente a medida que ainda sustenta uma problemática atual. A discussão é diretamente relacionada ao surgimento dos primeiro periódicos científicos, a consolidação de um Sistema de Comunicação Científica, o Science Citation Index (SCI) de Garfield, dentre outros fatores. Tal crise, juntamente com o aperfeiçoamento das novas tecnologias e o surgimento da internet, compôs os alicerces do ALi.

A crise dos periódicos consistiu num aumento do preço dos periódicos científicos de tal magnitude (PANTICH; MICHALAK, 2005) que levou muitas das maiores bibliotecas universitárias a cancelar assinaturas no final dos anos 1990. Entre 1986 e 2003, por exemplo, o valor de assinatura dos periódicos aumentou nos Estados Unidos $215 \%$ em média, contra uma inflação medida pelo Consumer Price Index de apenas $68 \%$. Esses aumentos se deram no quadro de uma concentração sem precedentes da propriedade dos periódicos no mercado internacional (ORTELLADO, 2008).

A maioria dos bibliotecários das instituições de pesquisa, segundo Okerson $(1991)^{27}$, acredita que esse sistema de publicação acadêmica não irá sobreviver sem, no mínimo, uma alteração radical nesse modelo, uma espécie de "renascimento" em outros moldes. Nesse sentido, a

\footnotetext{
${ }^{26}$ Salienta-se, aqui, que mesmo compondo a frase gramaticalmente no passado tal ciclo ainda é "visível" na estrutura atual apesar de todos os esforços, por parte do Movimento de Acesso Livre, no sentido contrário. Kuramoto (2006) diz: "Embora essa crise tenha começado em meados dos anos 80, ainda hoje não existe nenhuma solução definitiva. Com as tecnologias da informação e da comunicação, surge a iniciativa de arquivos abertos (Open Archives Initiative), a qual define um modelo de interoperabilidade entre bibliotecas $e$ repositórios digitais, possibilitando alternativas para a comunicação científica. Ao mesmo tempo, consolida-se o movimento em favor do acesso livre à informação cientifica em todo o mundo, pelos grandes editores ou publishers, por meio de propostas de ações que possam viabilizar essa iniciativa. Essas são as bases da proposta de um novo modelo para intensificar e consolidar o registro e a disseminação da produção científica brasileira, assim como do acesso à informação científica". Disponível em: <http://www.scielo.br/pdf/ ci/v35n2/a10v35n2.pdf>, acessado em 20 de outubro de 2009.

${ }^{27}$ OKERSON, Ann. 1991. Back to Academia? The Case for American Universities to Publish Their Own Research. Disponível em: <http://www.library.yale.edu/ okerson/case.html>, acessado em 1 de julho de 2009.
} 
autora cogita que uma alternativa a longo prazo seria que as próprias Universidades fossem autônomas no sentido de agirem como editores, visto que, mais de $70 \%$ dos artigos científicos são produzidos nessas instituições. Okerson convida, então, o leitor a compreender quais são os "principais sintomas" de tais propostas. Para tanto aborda a economia (de acordo com a Association of Research Libraries os preços dos livros e periódicos acadêmicos aumentou mais de $400 \%$ entre 1972 e 1988), o crescimento da literatura (John Naisbitt ${ }^{28}$ nos diz que a informação duplica a cada 5,5 anos) e as rápidas mudanças provocadas pelas tecnologias da informação (OKERSON; 1991).

Com o início da internet a questão do texto científico extrapolou a mera oportunidade de recuperação imediata do seu respectivo conteúdo e avançou no sentido de desestabilizar todo o processo de comunicação científica. Harnad $(1990)^{29}$ já afirmava, naquela ocasião, que estávamos atravessando uma "revolução comparável à ocasionada pela invenção da imprensa" reestruturando, inclusive, a prossecução do conhecimento. Os "efeitos potenciais dessa interatividade global” só não eram tão radicais em função de 8 obstáculos elencados e contra-argumentados pelo autor. A visão visionária do pesquisador naquele momento é registrada como algo de grande valia no sentido de contribuir para a construção de alicerces mais palpáveis ao ALi. Lembrando, aqui, que estamos nos referindo aos últimos 20 ou 25 anos. Nesse sentido, salienta-se, que uma parte significativa das explicações conceituais derivam do momento histórico no qual tais parâmetros são desenvolvidos. É quase impossível descontextualizar o objeto do sujeito quando das análises interpretativas que emergem do "olhar estrangeiro" sobre algo pertencente ao passado. Até que ponto essas "velhas maneiras de pensar" ainda são evidentes? Qual o grau de percepção dos autores sobre os processos emergentes da comunicação e da publicação científica? A adesão às novas estruturas só se confirma a partir dos mandatos ${ }^{30}$ ? Harnad (1990) já respondia: “Old ways of thinking will not be corrected by futuristic proposals; only convincing demonstrations of the potential power, productivity and scope of Scholarly Skywriting will capture the scientific community's allegiance and participation".

\footnotetext{
${ }^{28}$ Informações sobre o mesmo podem ser obtidas em $<$ http://www.naisbitt.com/biography.html $>$.

${ }^{29}$ HARNAD, Stevan. 1990. Scholarly Skywriting and the Prepublication Continuum of Scientific Inquiry. Disponível em: <http://cogprints.org/1581/0/harnad90.skywriting.html>, acessado em 12 de fevereiro de 2009.

${ }^{30}$ Resoluções criadas, por exemplo, pelas Instituições de Ensino, que obrigam o pesquisador a depositar/arquivar os resultados das suas pesquisas em repositórios.
} 
A "proposta subversiva" de Harnad é conhecida também por "Self Archiving Initiative" e está centrada na disponibilização dos textos pre-prints como forma de romper com as barreiras comerciais das grandes editoras direcionando, ao mesmo tempo, o enfoque para os arquivos abertos disponíveis na internet. Uma obra fundamental nessa discussão é "Scholarly Journals at the Crossroads: a Subversive Proposal for Electronic Publishing - an Internet Discussion about Scientific and Scholarly Journals and Their Future"31, de Okerson e O'Donnell (1995).

Mas, quais são os benefícios do Acesso Aberto? O que os teóricos dizem a respeito? E o posicionamento contrário? Existe resistência ao Movimento? A obviedade da expansão e/ou aceleração da ciência em função da difusão do conhecimento disponível em acesso aberto é uma prerrogativa básica. A partir desta, podemos prever uma maior visibilidade da produção científica e, consequentemente, um crescimento nas métricas e citações gerando, por conseguinte, reverberações positivas na sociedade. Será? Alguns autores sugerem que "sim", outros que "não" e dois trabalhos de revisão interessantes nessa perspectiva, dos "prós e contras" das citações e dos impactos mediante o acesso aberto, são " Open Access Citation Advantage: An Annotated Bibliography ", " "The effect of open access and downloads ('hits') on citation impact: a bibliography of studies ${ }^{33,}$.

Percebe-se, pois, que dentre as diversas problemáticas em torno da temática do acesso aberto a questão do "impacto" e da "visibilidade", principalmente dos artigos, é algo recorrente. Podemos citar, ainda, o estudo de Lawrence (2001) "Free online availability substantially increases a paper's impact "34, no qual são analisados 119.924 artigos de conferência em informática e áreas correlatas e o resultado é: para artigos offline o número de citações é 2.74 e para os que estão online 7.03 uma diferença de quase $257 \%$ e um outro trabalho sobre "The effect of Open Access on Citation Impact ${ }^{, 35}$, Tim Brody, Heinrich Stamerjohanns, Stevan

\footnotetext{
${ }^{31}$ OKERSON, Ann Shumelda; O’DONNELL, James J. 1995. A Subversive Proposal for Electronic Publishing: An Internet Discussion about Scientific and Scholarly Journals and Their Future. Disponível em: $<$ http://www.arl.org/bm doc/subversive.pdf >, acessado em 22 de agosto de 2009.

${ }^{32}$ WAGNER, A Ben. Open Access Citation Advantage: An Annotated Bibliography. In: Science Librarian. 2010. Disponível em: $<\mathrm{http}: / /$ www.istl.org/10-winter/article2.html>, acessado em 22 de agosto de 2009.

${ }^{33}$ The effect of open access and downloads ('hits') on citation impact: a bibliography of studies. 2010. Disponível em: <http://opcit.eprints.org/oacitation-biblio.html>, acessado em 1 de abril de 2009.

${ }^{34}$ LAWRENCE, Steven. 2001. Free online availability substantially increases a paper's impact. Nature. vol. 411, nr. 6837, p. 521.Disponível em: <http://www.nature.com/nature/debates/e-access/Articles/lawrence.html>, acessado em 19 de março de 2009.

${ }^{35}$ HARNAD, Stevan; BRODY, Tim; STAMERJOHANNS, Heinrich; GINGRAS, Yves; OPPENHEIM; Charles. et al. s.d. The effect of Open Access on Citation Impact. Disponível em: http://users.ecs.soton.ac.uk
} 
Harnad, Yves Gingras e Charles Oppenheim, com base nos dados da ISI Web of Science e do ArXiv, apontam que os papers disponíveis em acesso aberto são citados de 2.5 a 5.8 vezes mais que os que se encontram em posição contrária.

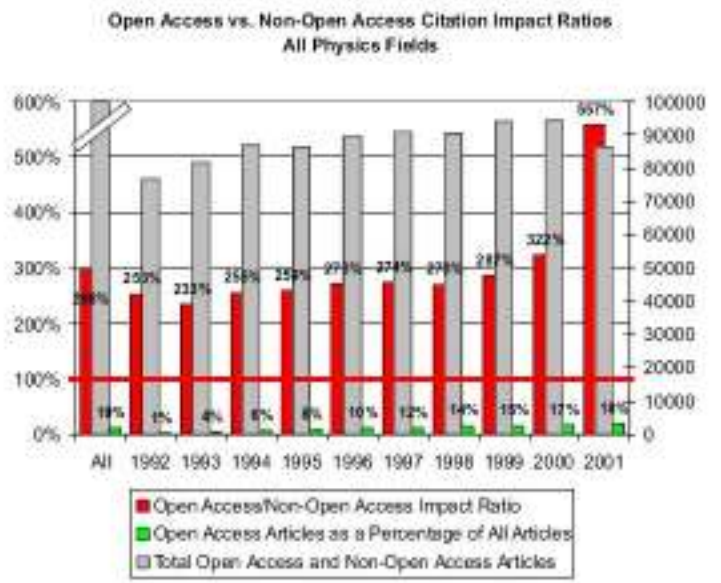

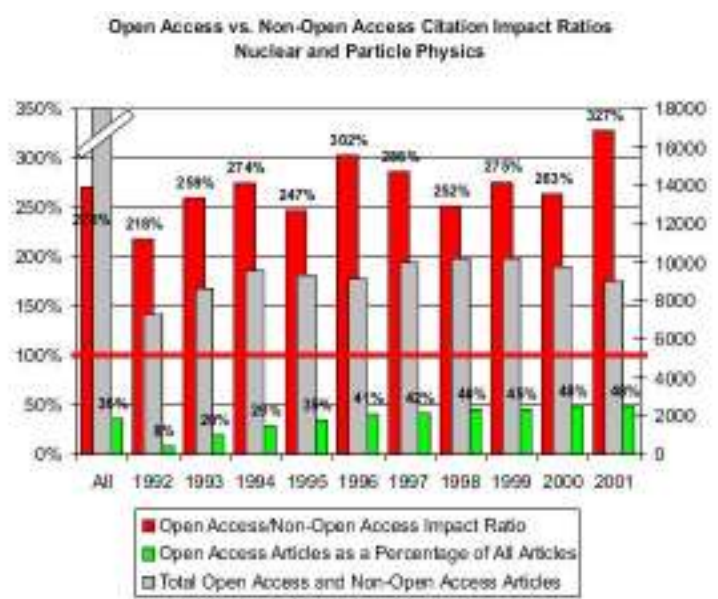

Figura 2 - The Effect of Open Access on Citation Impact

Fonte: Disponível em: <http://users.ecs.soton.ac.uk/harnad/Temp/OA-TAadvantage.pdf $>$, acessado em 29 de novembro de 2009.

\section{Vias Verde e Dourada}

Dois "marcos regulatórios" foram supracitados: de um lado a Declaração de Budapeste (Budapest Open Access Initiative) e de outro a de Berlim (Berlin Declaration). A iniciativa primeira, em certo sentido, transfigura as problemáticas vivenciadas naquele momento e promove o engajamento para ações futuras. Justifica o Open Acess como um "bem público", nesse caso, sem precedentes, para que o conhecimento científico possa ser compartilhado, "accelerate research, enrich education, share the learning of the rich with the poor and the poor with the rich, make this literature as useful as it can be, and lay the foundation for uniting humanity in a common intellectual conversation and quest for knowledge ". A

/harnad/Temp/OA-TAadvantage.pdf, acessado em: 19 de março de 2009. Disponível em: $<$ http://www.ecs.soton.ac.uk/ harnad/Temp/OA-TAadvantage.pdf>, acessado em 19 de março de 2009.

${ }^{36}$ Budapespt Open Access Initiative. 2001. Disponível em: <http://www.soros.org/openaccess/read.shtml>, acessado em 15 de maio de 2009 
literatura disponível em acesso aberto, mesmo restrita, já designava para um possível equilíbrio econômico, quando da sua gestão, além disso, o texto da Iniciativa aponta, por um lado, para os benefícios advindos do movimento em questão aos autores como, por exemplo, a maior visibilidade de sua produção e, por outro, clama por apoio as instituições com o propósito de "abrirem o acesso para o resto desta literatura" e romperem com as barreiras existentes, principalmente, os preços.

O documento referencia os artigos científicos avaliados por pares (peer-reviewed), mas amplia o leque de alternativas revelando, também, a importância da exposição de outros arquivos que podem ser disponibilizados via internet, ocasionando, por consequência, comentários dos colegas e/ou alertas relacionados aos seus resultados de investigação. A única restrição para a reprodução e distribuição de qualquer texto é, obviamente, a garantia dos direitos autorais (copyright) do pesquisador envolvido.

O texto da Declaração de Berlim sobre Acesso Livre ao Conhecimento nas Ciências e Humanidades ${ }^{37}$ também segue a mesma natureza:

\section{Objetivos:}

A nossa missão de disseminar o conhecimento estará incompleta se a informação não for tornada rapidamente acessível e em larga escala à sociedade. Novas possibilidades de difusão do conhecimento, não apenas através do método clássico, mas também, e cada vez mais, através do paradigma do acesso livre via internet devem ser apoiadas. Nós definimos o acesso livre como uma fonte universal do conhecimento humano e do patrimônio cultural que foi aprovada pela comunidade científica.

Para concretizar esta visão de uma representação global e acessível do conhecimento, a web do futuro tem de ser sustentável, interativa e transparente. Conteúdos e ferramentas de software devem ser livremente acessíveis e compatíveis.

\section{Definição de uma contribuição em Acesso Livre:}

Idealmente, o estabelecimento do acesso livre como um procedimento vantajoso requer o empenho ativo de todo e qualquer indivíduo que produza conhecimento científico ou seja detentor de patrimônio cultural. Contribuições em acesso livre incluem resultados de investigações científicas originais, dados não processados e metadados, fontes originais, representações digitais de materiais pictóricos e

\footnotetext{
${ }^{37}$ BERLIN Declaration on Open Access to Knowledge in the Sciences and Humanities. 2003. Disponível em: http://oa.mpg.de/openaccess-berlin/berlindeclaration.html, acessado em 15 de maio de 2009.
} 
gráficos e material acadêmico multimédia.

As contribuições de acesso aberto devem satisfazer duas condições:

1. O autor e o detentor dos direitos de tais contribuições concedem para todos os usuários o direito livre e gratuito, irrevogável e mundial de acessar a obra e licenciam a sua cópia, uso, distribuição, transmissão e disposição pública e a elaboração e distribuição de obras derivadas em qualquer meio digital para qualquer propósito responsável, sujeito à atribuição adequada de autoria (os padrões comunitários continuarão a prover os meios para o cumprimento da atribuição adequada e responsável da obra publicada, como acontece agora), assim como o direito de fazer poucas cópias para o seu uso pessoal.

2. A versão completa do trabalho e todos os materiais complementares, incluindo a cópia da permissão citada acima (e portanto publicada) é depositada em formato eletrônico padrão em ao menos um repositório usando padrões técnicos adequados (tais como as definições do Open Archive) que é mantido por uma instituição acadêmica, sociedade científica, agência governamental ou outra instituição bem estabelecida que busca permitir o acesso aberto, a distribuição irrestrita, a interoperabilidade e o arquivamento de longo prazo ${ }^{38}$.

No âmbito da mesma declaração fica explícito a questão da promoção de um novo "paradigma de acesso aberto" que maximize as potencialidades da ciência e da sociedade. Para tal, a mesma aponta a necessidade de "progredirmos" no sentido de: "encorajar os nossos investigadores/bolseiros a publicar os seus trabalhos de acordo com os princípios do paradigma de acesso livre; encorajar os detentores de patrimônio cultural a apoiar o acesso livre através da disponibilização dos seus recursos na Internet; desenvolver meios e formas para avaliar contribuições em acesso livre e jornais on-line de forma a assegurar os padrões de qualidade e as boas práticas cientificas; advogar que a publicação em acesso livre seja reconhecida para efeitos de avaliação e progressão acadêmica; demonstrar o mérito intrínseco das contribuições para uma infra-estrutura de acesso livre pelo desenvolvimento de ferramentas de software, fornecimento de conteúdos, criação de metadados ou a publicação individual de artigos ${ }^{39,}$.

As diferenças entre esta e a primeira estão assentadas, principalmente no fato de uma ser

\footnotetext{
${ }^{38}$ Adaptação da versão portuguesa elaborada pelos Serviços de Documentação da Universidade do Minho (UMinho - Portugal). Disponível em: <http://repositorium.sdum.uminho.pt/about/DeclaracaoBerlim.htm>, acessada em 3 de novembro de 2009

${ }^{39}$ Adaptação da versão portuguesa elaborada pelos Serviços de Documentação da Universidade do Minho (UMinho - Portugal). Disponível em: <http://repositorium.sdum.uminho.pt/about/DeclaracaoBerlim.htm>, acessada em 3 de novembro de 2009.
} 
posterior em relação à outra, fortalecendo as idéias e/ou propostas da primeira. A de Berlin, no entanto, estende seu modelo ao conjunto dos resultados de Pesquisa e do Patrimônio Cultural. $\mathrm{Na}$ de Budapeste a assinatura pode ser individual e é feita via internet40; já a de Berlin só aceita assinaturas de instituições41.

Duas estratégias complementares são recomendadas pela Declaração de Budapeste: uma intitulada "via verde", na qual o acesso livre é proporcionado através do auto-arquivamento das pesquisas científicas por parte do autor em repositórios e a outra, denominada "via dourada", é referente aos periódicos científicos de acesso aberto.

No termo "via verde" está implícita a recomendação, por parte do Movimento, para que os autores auto-arquivem, principalmente seus artigos publicados em revistas científicas, nos repositórios das instituições acadêmicas que estão vinculados. Tal prerrogativa, no entanto, além de não ser cumprida de forma espontânea por nem $15 \%$ dos pesquisadores ${ }^{42}$, ainda conta com o agravante de, em certos países periféricos, como no caso do Brasil, não contarmos com tais repositórios em todas as Universidades - o que pode ser "contornado", segundo entrevista dada por Steven Harnad ${ }^{43}$ - através do depósito do respectivo trabalho em outros repositórios científicos disponíveis. Uma outra "solução", levantada pelo supracitado autor, são os "mandatos", ou seja, as "leis" ou "diretrizes básicas" que obrigam o auto-arquivamento dos artigos, imediatamente depois da confirmação do aceite do mesmo para publicação. Já a "vida dourada", de acordo com Stevan, é “muito lenta e incerta, e também representa um potencial problema para os autores-instituições que não puderem arcar com os custos dessas publicações. As publicações de ALi tornar-se-ão de muito baixo custo, uma vez que todas as revistas serão convertidas para o ALi, mas elas somente serão convertidas - e os preços cairão - se 100\% do ALi forem primeiro atingidos pela outra via do Acesso Livre: a 'Verde',".

\footnotetext{
${ }^{40} \mathrm{Http}: / /$ www.soros.org/openaccess/sign.shtml

${ }^{41}$ Entrando em contato com os responsáveis no email: open-access@mpdl.mpg.de

42،'OA Mandates: Across all countries and disciplines, 95\% of researchers report that they would comply with a self-archiving mandate from their funders and/or employers, and over $80 \%$ report that they would do so willingly. -- But only 15\% self-archive spontaneously, if it not mandated". HARNAD, Stevan. Optimizing and integrating Open Access Mandates. Disponível em: <http:/confoa08.sdum.uminho.pt/apresentacoes/ stevan_harnard_portugal.pdf $>$, acessado em 2 de fevereiro de 2009.

${ }^{43}$ Entrevista com Steven Harnad (versão Hélio Kuramoto). In: In: Bibli.: R. Eletr. Bibliotecon. Ci. Inf., Florianópolis, n. Esp., $1^{\mathrm{o}}$ sem. 2007. Disponível em: <http://www.periodicos.ufsc.br/index.php/ eb/article/viewFile/647/518>, acessado em 2 de setembro de 2009.
} 
Como recomendado pela Declaração de Berlim, o ROARMAP ${ }^{44}$ (Registry of Open Access Repository Material Archiving Policies) acompanha o crescimento do número de mandatos e repositórios e, consequentemente, do auto-arquivamento. Hoje temos 146 mandatos e 15 "total proposed mandates" 45 .

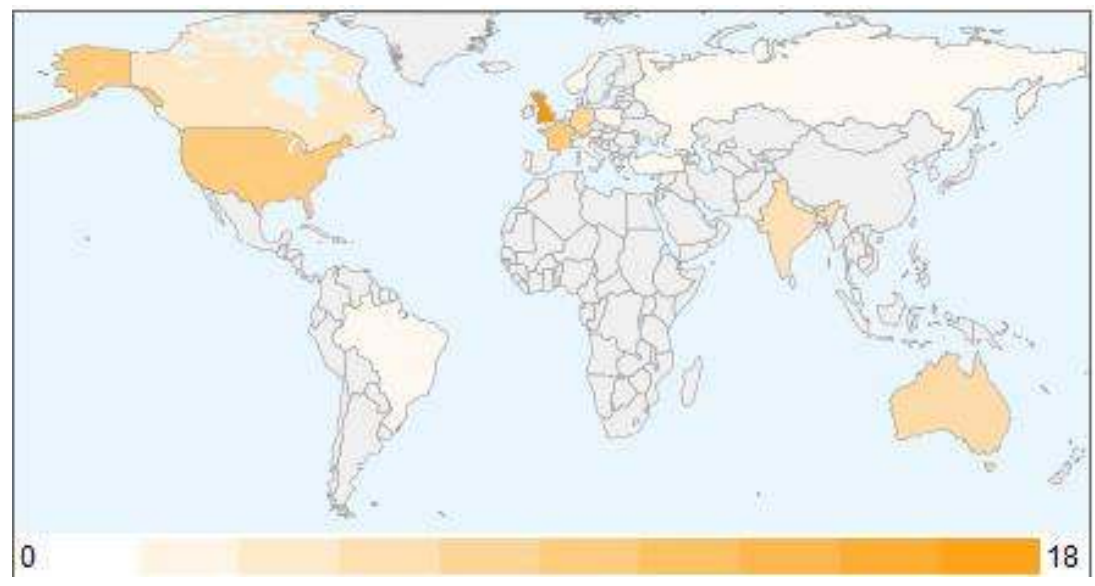

Figura 3 - Número de Mandatos

Fonte: ROARMAP. Disponível em: <http://www.eprints.org/openaccess/policysignup/>, acessado em 29 de novembro de 2009.

Os Repositórios Institucionais estão atrelados, na maioria das vezes, às Universidades e podem agrupar uma variedade enorme de documentos e formatos, desde teses até relatórios técnicos de eventos científicos, por exemplo. Segundo Rodrigues, "o movimento de criação de Repositórios Institucionais desenvolveu-se de forma significativa a partir de 2002, no entanto, a percentagem da produção cientifica mundial que recolhem ainda deve ser inferior a 10\%"46. A questão dos direitos autores (copyright), também segundo Rodrigues, "já não é um obstáculo importante ao desenvolvimento dos Repositórios Institucionais. Mais de 90\%

\footnotetext{
${ }^{44}$ ROARMAP (Registry of Open Access Repository Material Archiving Policies). Disponível em: $<$ http://www.eprints.org/openaccess/policysignup/>, acessado em 09 de novembro de 2009.

${ }^{45}$ ROARMAP. (Registry of Open Access Repository Material Archiving Policies). Disponível em: $<$ http://www.eprints.org/openaccess/policysignup/>, acessado em 29 de novembro de 2009.

${ }^{46}$ RODRIGUES, Eloy. Acesso Livre ao Conhecimento: a mudança do sistema de comunicação da ciência e os profissionais da informação. Disponível em: <http://repositorium.sdum.uminho.pt/handle/1822/670>, acessado em 13 de novembro de 2009.
} 
das revistas já permitem alguma forma de auto-arquivo"47.

Os indicadores dos repositórios ${ }^{48}$ podem ser obtidos no Registry of Open Access Repositories $(\text { ROAR })^{49}$. Nele encontramos 1.523 (hum mil quinhentos e vinte três) repositórios cadastrados em 09 de novembro de 2009, sendo 70 deles localizados no Brasil. O mesmo se encontra na quinta posição atrás somente dos EUA com 300 repositórios, Reino Unido com 151, Alemanha com 112 e Japão com 71. Com relação ao tipo de conteúdo: 851 deles são "Research Institutional or Departmental", 141 "Research Cross-Institucional", 135 de "ETheses", 111 de "E-Journal/Publication", 36 de "Database/A\&I Index", 22 de "Demonstration", 12 de "Learning and Teaching Objects" e 215 que estão classificados na categoria “Other". Percebe-se, pois, que quase 56\% dos repositórios estão nos centros de pesquisas institucionais ou departamentais. No Brasil 28 repositórios são classificados em "EJournal/Publication", 17 pontuados como "Other", 14 "Research Institucional or Departamental", 6 de "E-Theses", 3 de "Research Cross-Institutional" e 1 nas categorias "Database/A\&I Index" e "Learning and Teaching Objects".

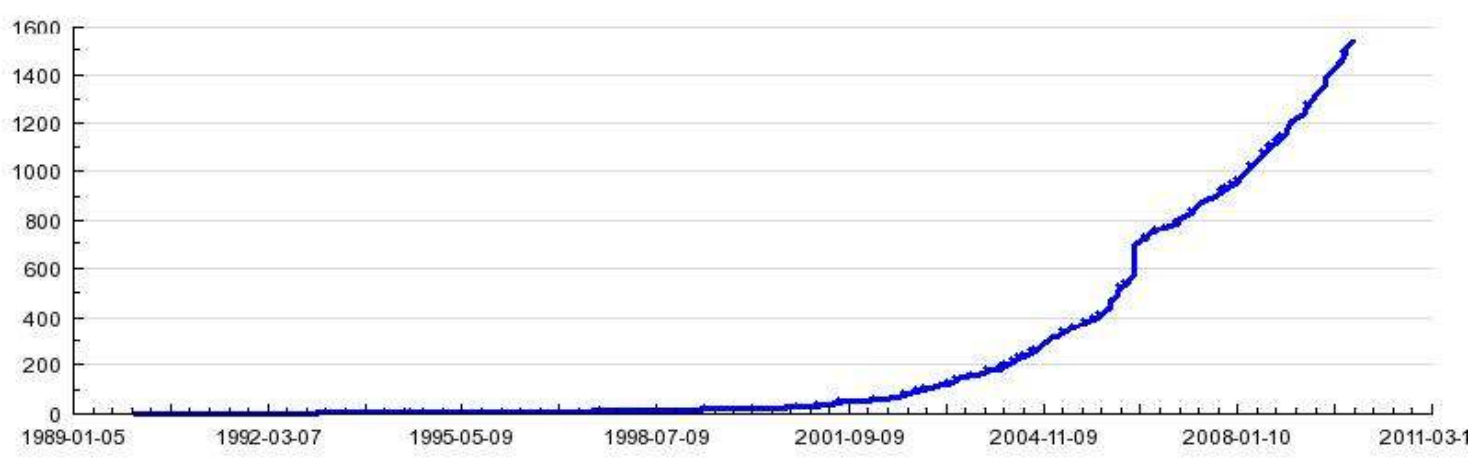

Figura 4 - Repositories Registered Over Time

Fonte: ROAR. Disponível em: <http://roar.eprints.org/index.php?action=generate_chart>, acessado em 29 de novembro de 2009.

\footnotetext{
${ }^{47}$ RODRIGUES, Eloy. Acesso Livre ao Conhecimento: a mudança do sistema de comunicação da ciência e os profissionais da informação. Disponível em: <http://repositorium.sdum.uminho.pt/handle/1822/670>, acessado em 13 de novembro de 2009.

48 “....] os provedores de dados e de serviços inscrevem os seus repositórios, indicando o pacote de software utilizado. Esse sítio possui uma metodologia de verificação de conformidade dos repositórios com o protocolo OAI-PMH, o qual possibilita o acompanhamento do crescimento dos repositórios, classificando-os por quantidade de registros, por país e por tipo de aplicação" (Kuramoto, 2006). Disponível em: $<$ http://www.scielo.br/pdf/ci/v35n2/a10v35n2.pdf>, acessado em 25 de maio de 2009.

${ }^{49}$ ROAR. Disponível em: <http://roar.eprints.org/>, acessado em 28 de novembro de 2009.
} 


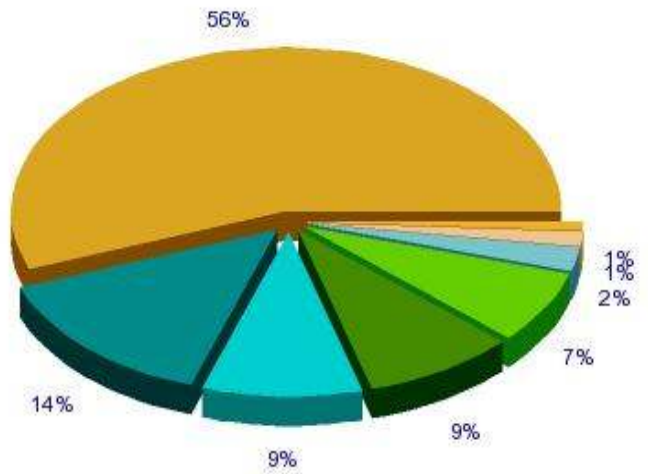

Research Institutional or Departmental (860)

Other (218)

Research Cross-Institutional (144)

e-Theses (138)

e-Journal/Publication (111)

Database/A\&I Index (36)

Demonstration (22)

Learning and Teaching Objects (13)

Figura 5 - Repositories by Content Type

Fonte: ROAR. Disponível em: <http://roar.eprints.org/index.php?action=generate_chart\&chart_type=pie>, acessado em 29 de novembro de 2009.

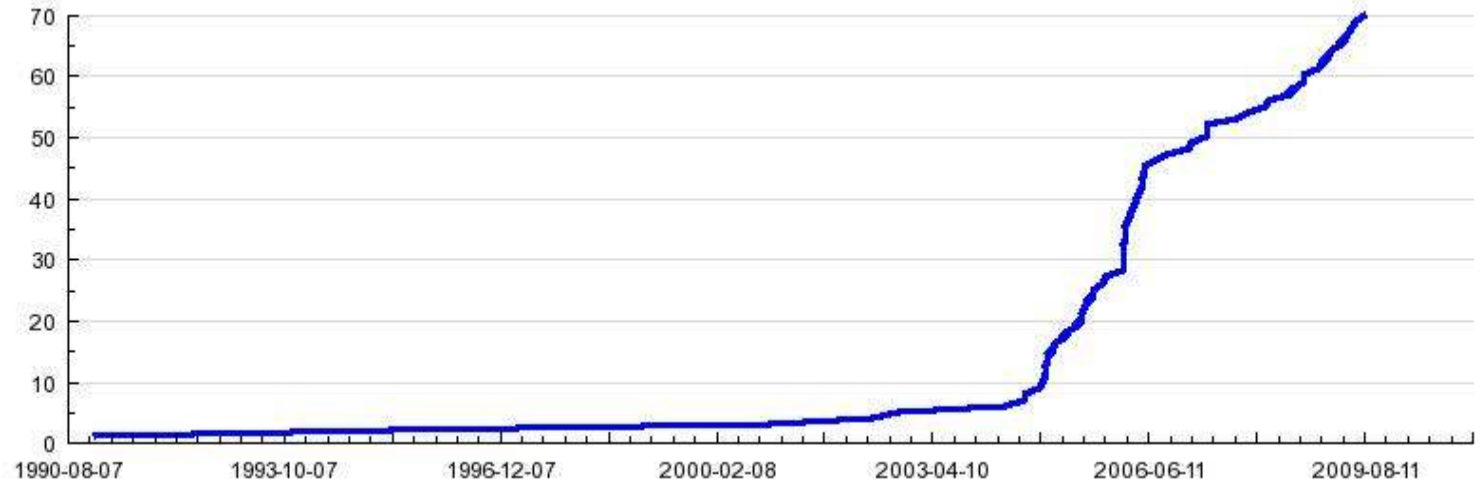

Figura 6 - Repositories Registered Over Time - Brasil

Fonte: ROAR. Disponível em: $<$ http://roar.eprints.org/index.php?action=generate chart\&q=\&country=br\& version $=\&$ type $=\&$ order $=$ name $\&$ submit $=$ Filter $>$, acessado em 29 de novembro de 2009. 


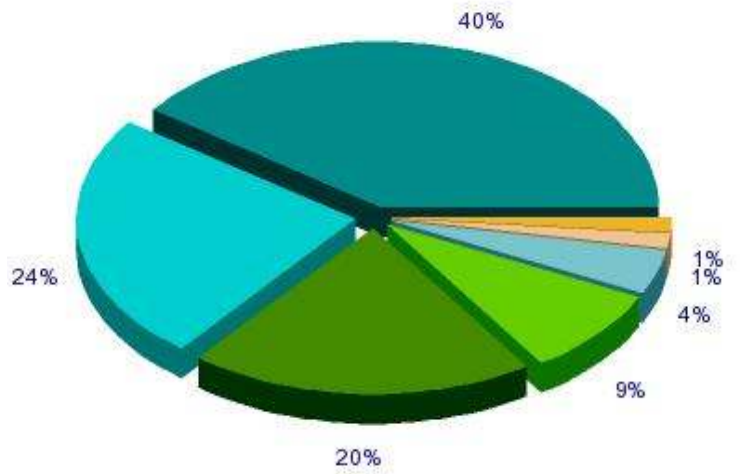

$20 \%$
e-Journal/Publication (28)

Other (17)

Research Institutional or Departmental (14)

e-Theses (6)

Research Cross-Institutional (3)

Database/A\&l Index (1)

Learning and Teaching Objects (1)

Figura 7 - Repositories by Content Type - Brasil

Fonte: ROAR. Disponível em: <http://roar.eprints.org/index.php?action=generate_chart\&q=\&country=br\& version $=\&$ type $=\&$ order $=$ name \&submit $=$ Filter\&chart type $=$ pie $>$, acessado em 29 de novembro de 2009.

$\mathrm{O}$ EPrints ${ }^{50}$ mesmo sendo o primeiro pacote de software para criação de Repositórios Institucionais em conformidade com o modelo estabelecido pela Open Access Iniciative encontra-se em segundo lugar na tabela de "System Software" com 348 ocorrências. Na primeira posição está o DSpace ${ }^{51}$ com 487 arquivos e em terceiro o Bepress ${ }^{52}$ com 89 . Em âmbito nacional, 49 repositórios utilizam outros softwares, 14 o DSpace, 4 o EPrints, 2 o ETD-db ${ }^{53}$ e 1 o Open Journal System ${ }^{54}$.

Após o estabelecimento do modelo de interoperabilidade pela OAi, a mais recente tecnologia para ALi são os pacotes de software livre escritos para criar arquivos em conformidade com esses padrões (também chamados repositórios: Repositórios Institucionais (RI) e Repositórios Centrais (RC)). O primeiro pacote de software para criação de RI em conformidade com o modelo estabelecido pela OAi, amplamente utilizado em todo o mundo é o Eprints. Associado ao Eprints existem também diretórios de repositórios institucionais em todo o mundo, e diretórios de

\footnotetext{
${ }^{50}$ Open Access and Institutional Repositories with EPrints. Disponível em: < http://www.eprints.org>, acessado em 09 de novembro de 2009.

${ }^{51}$ DSpace. Disponível em: <http://www.dspace.org/>, acessado em 09 de novembro de 2009.

${ }^{52}$ Bepress Legal Repository. Disponível em: <http://law.bepress.com/repository/>, acessado em 09 de novembro de 2009.

${ }^{53}$ Digital Library and Archives. Disponível em: <http://scholar.lib.vt.edu/ETD-db/index.shtml>, acessado em 09 de novembro de 2009.

${ }^{54}$ Open Journal Systems. Disponível em: <http://pkp.sfu.ca/?q=ojs >, acessado em 09 de novembro de 2009.
} 
políticas de ALi Institucionais. Um RI de Ali suportado por um mecanismo de busca baseado em citações, Citebase, foi criado. Ele navega por meio das citações, da mesma forma que o Google navega por meio de links. Ele tem ajudado também a abrir um novo campo na cientometria do ALi: navegando e avaliando as bases de dados contendo resultados de pesquisa de ALi, por meio de antigas e novas métricas do fator de impacto relativo aos resultados de pesquisa, tais como downloads, citações, cocitações, hubs/authorities2, cronometria, etc ${ }^{55}$.

E, na linha da "via dourada" é interessante observarmos a existência de 4.420 Periódicos eletrônicos com acesso aberto no Directory of Open Access Journals (DOAJ) ${ }^{56}$, sendo possível a realização de pesquisas no nível dos artigos em 1.687 deles. No serviço DOAJ estão inclusos 322.464 artigos. $^{57}$

\section{Considerações Finais}

Uma maior imersão sobre a cronologia do Acesso Aberto pode ser obtida consultando o documento publicado por Peter Suber na internet denominado "Timeline of Open Access Movement" $"$. Nele o autor remonta ao ano de 1966 quando o "Educational Resources Information Center (ERIC) launched by the U.S. Department of Education's Office of Educational Research and Improvement and the National Library of Education", perpassando, ainda, o lançamento do "The Open Citation Project (OpCit)" em 1999, para culminar "Congress passed, and the President signed, a spending bill mandating OA to research funded by the US National Institutes of Health (NIH)" em 26 de dezembro de 2008. Observa-se, porém, que o presente trabalho apresentou uma trilha teórica composta pelos viéses fundamentais do Acesso Aberto sem, no entanto, verticalizar no sentido de perpassar todas as entrelinhas das problemáticas apontadas. Como uma teia que comportou diferentes caminhos, em algumas ocasiões, as "direções foram só apontados" e cabe, agora, ao leitor,

\footnotetext{
${ }^{55}$ Entrevista com Steven Harnad (versão Hélio Kuramoto). In: In: Bibli.: R. Eletr. Bibliotecon. Ci. Inf., Florianópolis, n. Esp., $1^{\text {o }}$ sem. 2007. Disponível em: <http://www.periodicos.ufsc.br/index.php/eb/article/ viewFile/647/518>, acessado em 18 de outubro de 2009.

56 Directory of Open Access Journals. Disponível em: <http://www.doaj.org>, acessado em 09 de novembro de 2009.

57 Directory of Open Access Journals. Disponível em: <http://www.doaj.org>, acessado em 09 de novembro de 2009.

${ }^{58}$ SUBER, PETER. 2009. Timeline of Open Access Movement. Disponível em: http://www.earlham.edu/ peters /fos/timeline.htm>, acessado em 17 de abril de 2009.
} 
buscar de forma independente, fontes e links ${ }^{59}$ complementares e promover, no âmbito dos debates acadêmicos do Turismo, novas reflexões, inserções, diretrizes e teorias.

\section{Referências}

ARCHIVES OF AMERICAN SCIENTIFIC OPEN ACCESS FORUM. Disponível em: $<$ http://amsciforum.amsci.org/archives/American-Scientist-Open-Access-Forum.html $>$. Acessado em 2 de fevereiro de 2009.

BERLIN Declaration on Open Access to Knowledge in the Sciences and Humanities. 2003. Disponível em: http://oa.mpg.de/openaccess-berlin/berlindeclaration.html, acessado em 15 de maio de 2009.

BUDAPEST Open Access Initiative. 2001. Disponível em: <http://www.soror.org/openaccess/>. Acessado em: 19 de março de 2009.

BUTLER, Declan. 2001. Debates da Revista Nature. Disponível em: http://www.nature.com/nature/debates/e-access/Articles/ginsparg.html, acessado em 17 de agosto de 2009.

Directory of Open Access Journals. Disponível em: <http://www.doaj.org>, acessado em 09 de novembro de 2009.

EPRINTS. Disponível em: <http://www.eprints.org>. Acesso em: 02 de abril de 2009

HARNAD, S. Entrevista com Stevan Harnad. 2007. In: Bibli.: R. Eletr. Bibliotecon. Ci. Inf., Florianópolis, n. Esp., $1^{\circ} \mathrm{sem}$.

. Scholarly Skywriting and the prepublication continuum of scientific inquiry. 1990. Disponível em: http://cogprints.org/1581/0/harnad90.skywriting.html, acessado em 23 de julho de 2009.

Post-Gutenberg Galaxy: the fourth revolution in the means of production of knowledge. 1991. Disponível em: http://cogprints.org/1580/0/harnad91.postgutenberg.html, acessado em 23 de julho de 2009.

Learned inquiry and the net: the role of peer review, peer commentary and copyright. 1998. Disponível em: http://eprints.ecs.soton.ac.uk/2633/02/harnad98.toronto.learnedpub.html. Acessado em 19 de outubro de 2009. abril de 2009 .

(s.d). Open Access. Disponível em: <http://www.eprints.org/openaccess/>, acessado em 3 de

Optimizing and Integrating Open Access Mandates. 3a. Conferência sobre o Acesso Livre ao Conhecimento. Universidade do Minho. 15 a 16 de dezembro de 2008. Disponível em: $<$ http://confoa08.sdum.uminho.pt/programa.htm>, acessado em 2 de abril de 2009.

\footnotetext{
${ }^{59}$ A expressão links, aqui entendida como "conexões", "ligações" e "vínculos", é utilizada no sentido literal, ou seja, como estamos analisando uma área cuja base se articula à tecnologia, principalmente, através do acesso e do uso da internet como instrumento de coleta de dados, tal verbete se faz necessário.
} 
; BRODY, Tim; STAMERJOHANNS, Heinrich; GINGRAS, Yves; OPPENHEIM; Charles. et al. (s.d). "The effect of Open Access on Citation Impact". Disponivel em: $<$ http://www.ecs.soton.ac.uk/ harnad/Temp/OA-TAadvantage.pdf $>$, acessado em 23 de maio de 2009.

KURAMOTO, Hélio. Informação Científica: proposta de um novo modelo para o Brasil. In: Ciência da Informação.Brasília, v. 35, n.2, p.91-102, maio/ago. 2006. Disponível em: http://www.scielo.br/pdf/ci/v35n2/a10v35n2.pdf, acessado em 20 de outubro de 2009.

LARA, Marilda Lopes Ginez. Termos e conceitos da área de Comunicação e Produção Cientifica. In: POBLACIOM, Dinah Aguiar; WITTER, Geraldina Porto; SILVA, Fernando Modesto da (org). Comunicação e Produção Científica: contexto, indicadores, avaliação. São Paulo: Angellara, 2006.

LAWRENCE, Steven. 2001. Free online availability substantially increases a paper's impact. Nature. vol. 411 , nr. 6837 , p. 521

MANIFESTO BRASILEIRO DE APOIO AO ACESSO LIVRO À INFORMAÇÃO CIENTÍFICA. Disponível em: http://kuramoto.files.wordpress.com/2008/09/manifesto-sobre-o-acesso-livre-ainformacao-cientifica.pdf, acessado em 11 de outubro de 2009.

OKERSON, Ann Shumelda; O’DONNELL, James J. 1995. A Subversive Proposal for Electronic Publishing: An Internet Discussion about Scientific and Scholarly Journals and Their Future. Disponível em: http://www.arl.org/bm doc/subversive.pdf, acessado em 22 de agosto de 2009.

; 1991. Back to Academia? The Case for American Universities to Publish Their Own Research. Disponível em: http://www.library.yale.edu/ okerson/case.html, acessado em 22 de agosto de 2009.

ORTELLADO, Pablo. As políticas nacionais de acesso a informação científica. In: Liinc em Revista, v.4, n.2, setembro 2008, Rio de Janeiro, p. 186-195. Disponível em: http://revista.ibict.br/liinc/index.php/liinc/article/viewFile/268/168, acessado em 19 de outubro de 2009.

ROARMAP (Registry of Open Access Repository Material Archiving Policies). Disponível em: $<\mathrm{http}: / /$ www.eprints.org/openaccess/policysignup/>, acessado em 16 de maio de 2009.

RODRIGUES, ELOY. 2004. Acesso Livre ao Conhecimento: a utopia e a realidade. disponível em: $<$ http://repositorium.sdum.uminho.pt/handle/1822/4942>, acessado em 17 de março de 2009.

2004b. Acesso Livre ao Conhecimento: a mudança do sistema de comunicação da ciência e os profissionais de informação. In: Cadernos de Biblioteconomia Arquivística e Documentação. Portugal: Lisboa. Associação Portuguesa de Bibliotecários, Arquivistas e Documentalistas. Número 01, 2004, p. 24-35

SOMPEL, Herbert van de; LAGOZE, Carl. The Santa Fé Convention of the Open Archives Initiative. D-Lib Magazine, v. 6, n. 2, Feb. 2000. Disponível em: <http://www.dlib.org/dlib/february00/ vandesompel-oai/02vandesompel-oai.html>, acessado em 10 de setembro de 2009.

SUBER, PETER. 2009. Timeline of Open Access Movement. Disponível em: $<$ http://www.earlham.edu/ peters/fos/timeline.htm>, acessado em 17 de abril de 2009. 
2007. Open Access Overview: Focusing on open acces to peer reviewd research articles and their preprints. Disponível em: http://www.earlham.edu/ peters/fos/overview.htm, acessado em 3 de abril de 2009.

The Santa Fe Convention for the Open Archives Initiative. Disponível em: http://www.openarchives.org/sfc/sfc_entry.htm, acessado em 10 de setembro de 2009.

WEITZEL, Simone da Rocha. Fluxo da Informação Científica. In: POBLACIOM, Dinah Aguiar; WITTER, Geraldina Porto; SILVA, Fernando Modesto da (org). Comunicação e Produção Científica: contexto, indicadores, avaliação. São Paulo: Angellara, 2006.

Recebido em: 26/04/2010

Aprovado em: 12/08/2010 